\title{
Surgical approaches and strategies for skull base chordomas
}

\author{
Norihiko Tamaki, M.D., Tatsuya Nagashima, M.D., Kazumasa Ehara, M.D., \\ YasuhiKo MOTOOKa, M.D., AND KanaK Kanti BARUa, M.D.
}

Department of Neurosurgery, Kobe University School of Medicine, Kobe, Japan

\begin{abstract}
Object. The management of chordomas involving the skull base continues to present a number of treatment-related problems. Recently, both radical resection and charged-particle irradiation or stereotactic radiosurgery have reportedly been found effective for tumor control and for promoting a better quality of life in patients. In this article the authors analyzed the outcomes in 17 patients with skull base chordomas who were surgically treated at Kobe University Hospital between 1972 and 2000.

Methods. Preoperative radiological examinations included magnetic resonance imaging, computerized tomography, angiography, and balloon occlusion test of the internal carotid artery. Among the various surgical approaches used to remove the tumor were the frontoorbitozygomatic, transmaxillary, transcondylar, transsphenoidal, and the transbasal. Total removal was achieved in two (12\%), near-total removal in three (18\%), subtotal removal in nine (52\%), and partial removal in three patients (18\%). Since 1990, chordomas have been radically resected via various skull base approaches; the combined total or near-total removal rate has been $80 \%$ in this period. Radical removal of the tumors has not led to an increased risk. At the final follow-up review (mean 59.5 month), 75\% of the patients were still alive, and $25 \%$ had died of chordoma recurrence. The overall recurrence-free survival rate was $82 \%$ at 3 years and $51 \%$ at 5 years. The 5-year recurrence-free survival rate in the five patients who underwent the operation during the past decade was $77 \%$ (mean follow up of 5.2 years). In two patients with recurrent tumors who underwent radiosurgery, no evidence of tumor regrowth was demonstrated at 3 years posttreatment.

Conclusions. The authors suggest that for the treatment of skull base chordomas radical resection is a key factor for longer survival and improved quality of life. Patients with sufficiently small tumors, which show a favorable configuration and location, can be suitable candidates for stereotactic radiosurgery.
\end{abstract}

\section{KEY WORDS • chordoma • microsurgery • radiosurgery • magnetic resonance imaging}

Cranial chordoma is a rare tumor that arises from the remnant of the notochord. Approximately $35 \%$ of chordomas occur in the sphenooccipital region and mostly involve the clivus, petrous apex, cavernous sinus, foramen magnum, and sphenoethmoidal areas. ${ }^{19}$ Because each region is associated with distinct surgical problems, the optimum treatment remains controversial. ${ }^{5}$ Much of this controversy results from the fact that, although a number of these tumors grow slowly, surgery has rarely been curative. Although chordomas are not histologically malignant, they are locally invasive and clinically malignant. ${ }^{29}$ The estimated overall survival rate is reported to be $51 \%$ and $35 \%$ at 5 years and 10 years, respectively. ${ }^{15}$ Because tumor recurrence is the major cause of death, ${ }^{6}$ the RFS rate is an important indicator of successful treatment. Furthermore, QOL is a very important consideration because some patients may live for many years even while harboring growing tumors. Our current treatment of these tumors

Abbreviations used in this paper: $\mathrm{CCJ}=$ craniocervical junction; $\mathrm{CSF}=$ cerebrospinal fluid; $\mathrm{CT}=$ computerized tomography; $\mathrm{ICA}=$ internal carotid artery; $\mathrm{MR}=$ magnetic resonance; $\mathrm{QOL}=$ quality of life; RFS = recurrence-free survival. consists of extensive resection of the tumor via various skull base approaches followed by conventional radiotherapy, and/or stereotactic radiosurgery. The dura mater is preserved or reconstructed to protect the intradural neural and vascular structures against recurrent tumors. Any adjacent abnormally appearing bone is removed. In this article, we review the treatment of 17 patients with skull base chordomas and discuss the radical resection of the tumors via various skull base approaches.

\section{CLINICAL MATERIAL AND METHODS}

\section{Patient Population}

Seventeen patients including 10 women and seven men with skull base chordomas were treated at Kobe University School of Medicine between 1972 and 2000. In three patients the lesion was diagnosed as chondroid chordoma. Those with chondrosarcomas were not included in this study. The mean age of these patients was 47 years (range 17-70 years). The most frequent initial symptoms were double vision (68\%) and headache (18.8\%). Two patients suffered from visual disturbance, and one patient with a tumor located at the CCJ reported experiencing 
neck pain. Except for one patient who was examined angiographically, all patients underwent neuroradiological examinations including MR imaging or CT scanning during the pre- and postoperative period. The examinations consisted of sagittal, axial, and coronal $\mathrm{T}_{1}$-weighted MR studies, with and without gadolinium enhancement, and $\mathrm{T}_{2}$-weighted MR studies. A bone-window $\mathrm{CT}$ scan and three-dimensional CT scan were obtained to demonstrate the extent of bone destruction.

The multidirectional extent of the tumor invasion made topographic classification difficult. All tumors had invaded the clivus, $10(58 \%)$ invaded the cavernous sinus, and one $(6 \%)$ invaded the CCJ. Using Al-Mefty's classification system, ${ }^{3} 13$ tumors were classified as Type II (tumors that extend over two or more contiguous areas of the skull base and can be radically resected via a single skull base approach) and four were as Type III (those that extend over several contiguous compartments of the skull base and require two or more skull base approaches). When the tumor involved the ICA, the sufficiency of collateral circulation was evaluated by measuring the cerebral blood flow with single-photon emission CT scanning and a carotid artery occlusion test.

Eight patients underwent follow up for more than 5 years and four for more than 10 years; the median followup duration was 4.9 years (range 0.6-18 years). Sixteen patients underwent follow-up MR imaging examination, and one underwent follow-up CT scanning. The patients' functional status was updated by direct examination or by obtaining information via telephone interview. Survival and RFS rates were determined by performing KaplanMeier analysis.

\section{Surgical and Radiation Treatment}

Fourteen patients underwent their initial operation at our institution. In twelve patients the tumor was removed via a single approach and in four via two approaches. The routes consisted of the transsphenoidal approach and the frontotemporal approach in four patients each; the combined transsphenoidal and frontoorbitozygomatic or frontoorbital approach in two patients; and the transoral approach, frontobasal approach, transmaxillary approach, transcondylar approach, suboccipital approach, the combined transsphenoidal-frontotemporal approach and the combined transoral-frontobasal approach in one patient each. In one patient an iliac bone graft was used for craniocervical stabilization after tumor resection. In all cases the extent of tumor resection was determined on the basis of operative reports and postoperative radiological studies. Total removal was defined as the absence of visible tumor intraoperatively and on postoperative radiological studies; near-total removal as questionable remnants observed intraoperatively or on postoperative radiological studies; subtotal removal as at least $90 \%$ excision of the tumor; and partial removal as less than $90 \%$ removal.

Postoperatively 13 patients (76\%) underwent conventional radiotherapy (range 40-69 Gy, average 50.8 Gy), and the two patients in whom recurrent tumors have been identified since 1990 underwent stereotactic radiosurgery (10-12 Gy delivered to the $50 \%$ isodose line). The treatment dose was selected by the neurosurgeon in conjunction with a radiation oncologist.

\section{RESULTS}

Total tumor removal was achieved in two (12\%), neartotal removal in three (18\%), subtotal removal in nine $(53 \%)$, and partial removal in three patients (18\%). Since 1990, chordomas have been radically removed using various skull base techniques, resulting in the total or near-total removal of tumor in four patients (80\%). In the patients who underwent our initial operation, no surgeryrelated death and minimal morbidity were demonstrated. One permanent surgery-related neurological deficit (third and sixth cranial nerve deficit) occurred. Temporary surgery-related complications included diabetes inspidus (one patient), fifth cranial nerve palsy (one patient), sixth cranial nerve palsy (two patients), lower cranial nerve palsy (one patient), and gastrointestinal bleeding (one patient). A higher risk was not associated with patients undergoing aggressive initial surgery. On the other hand, in those who had undergone previous operations and radiotherapy there was a higher risk of mortality and morbidity. One patient with a recurrent parasellar chordoma involving perforator vessels of the ICA died of cerebral infarction within 3 months of the operation.

At the last follow-up examination (mean 59.5 months) of the 16 patients who had been followed for more than 6 months, $12(75 \%)$ were alive and four had died of chordoma recurrence. No evidence of residual tumor was observed at 7 years and 9 years after the initial operations in two patients, respectively. Recurrence of the tumor was demonstrated in five patients, three of whom underwent reoperation and two of whom underwent radiosurgery. The mean interval between the first and second treatment for all five patients with recurrent tumors was 6.6 years ( 3 , $3,4,5$, and 18 years, respectively). In the two patients with recurrent tumors treated by radiosurgery no evidence of tumor regrowth was demonstrated 3 years postradiosurgery. The other three patients harbored residual tumors.

Survival analysis indicated that RFS was $82 \%$ at 3 years and $51 \%$ at 5 years. Overall survival rates were $83 \%$ at 3 years and $64 \%$ at 5 years. The overall survival curve showed a reduction during the first 5 years after surgery, after which stabilization occurred (Fig. 1). Five patients in whom radical resection was performed over of the past decade were followed for 5.2 years (range $0.5-10$ years). All of them survived and experienced good QOL, although one had to undergo stereotactic radiosurgery for a small recurrent tumor. Five-year RFS in patients who underwent the operation during the past decade was $77 \%$. We highlight the reports of the four patients who underwent radical resection of a tumor invading the skull base and of the patient who underwent stereotactic radiosurgery for a recurrent tumor.

\section{ILLUSTRATIVE CASES}

\section{Case 1}

This 48-year-old woman who presented with visual disturbance was found to harbor a parasellar chordoma. Magnetic resonance imaging revealed invasion of the bilateral ICAs, the upper two thirds of the clivus, the sella turtica, the sphenoid sinus, and the suprasellar region (Fig. 2 upper left and right). She underwent a near-total removal 


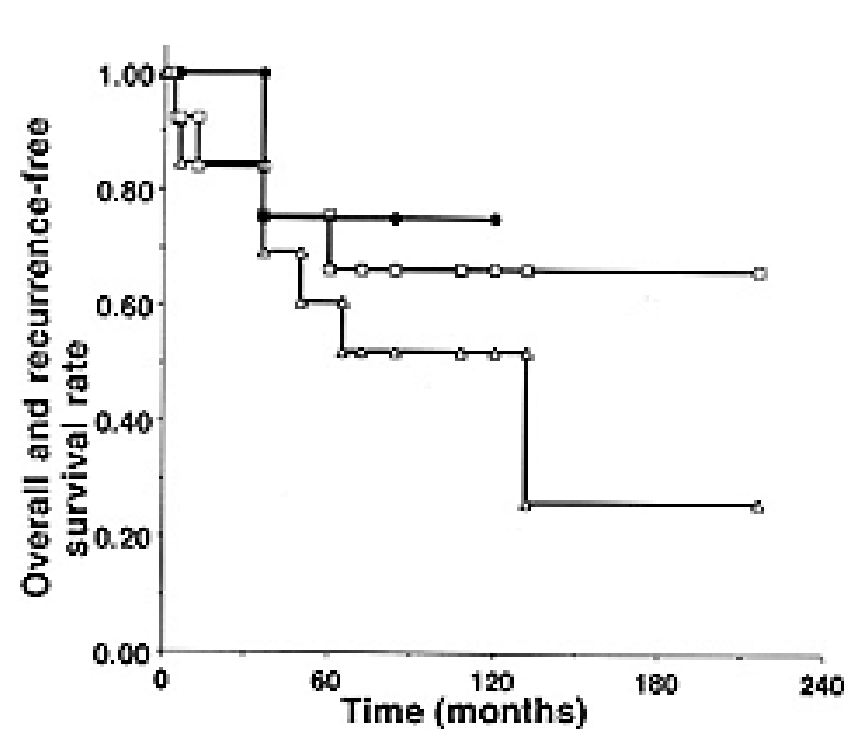

Fig. 1. Graph showing overall survival and RFS curves. Open square: overall survival rate for all 17 patients; open triangle: RFS rate for all 17 patients; closed circle: RFS rate for the patients treated during the past decade.

of the tumor via the frontobasal extradural approach (Fig. 2 lower left and right). Postoperatively she underwent conventional radiotherapy (a 60-Gy dose). Her visual disturbance improved, and she was living independently 10 years after the operation without any tumor recurrence.

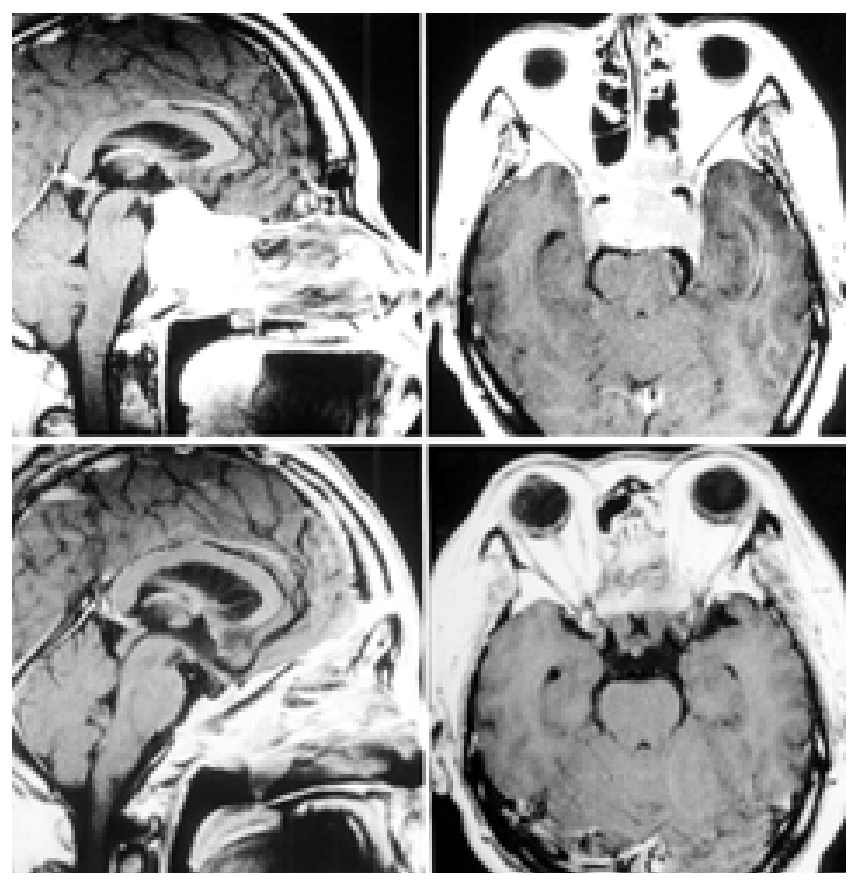

Fig. 2. Case 1. Gadolinium-enhanced MR images. Upper Left: Preoperative sagittal image revealing a large mass in the upper clivus, the sphenoid sinus, and suprasellar region. Upper Right: Preoperative axial image demonstrating bilateral cavernous sinus invasion. Lower Left: Postoperative sagittal image revealing no residual tumor 3 years after the operation. Lower Right: Postoperative axial image demonstrating no residual tumor in the cavernous sinuses.

\section{Case 2}

This 57-year-old man with right-sided visual disturbance was found to have a huge parasellar chordoma. Suprasellar extension, cavernous sinus invasion, and encasement of the ICA were revealed on MR imaging (Fig. 3 upper left and right). He underwent near-total removal of the tumor by means of staged operations via a combined transsphenoidal and the frontoorbitozygomatic transcavernous approach. The intracavernous ICA was preserved by dissecting it from the tumor. The defect left after tumor removal was filled with autologous fat and covered with a vascularized pericranial flap (Fig. 3 lower left and right). Lumbar CSF drainage was performed for 7 postoperative days, and the patient was treated with postoperative conventional irradiation (50 Gy). Postoperatively, his visual disturbance improved, but mild third and sixth cranial nerve palsy appeared. No tumor growth and no further progression of symptoms were observed 6 months later.

\section{Case 3}

This 17-year-old young man with left-sided hearing disturbance was found to have a midclival tumor, as demonstrated on MR imaging (Fig. 4 left). The tumor compressed the brainstem dorsally. Magnetic resonance imaging demonstrated that the tumor had reached the posterior clinoid process and the lower part of the clivus. The patient underwent gross-total removal of the tumor via the transmaxillary approach. Abnormally appearing bone near the tumor was drilled away. Postoperatively, the

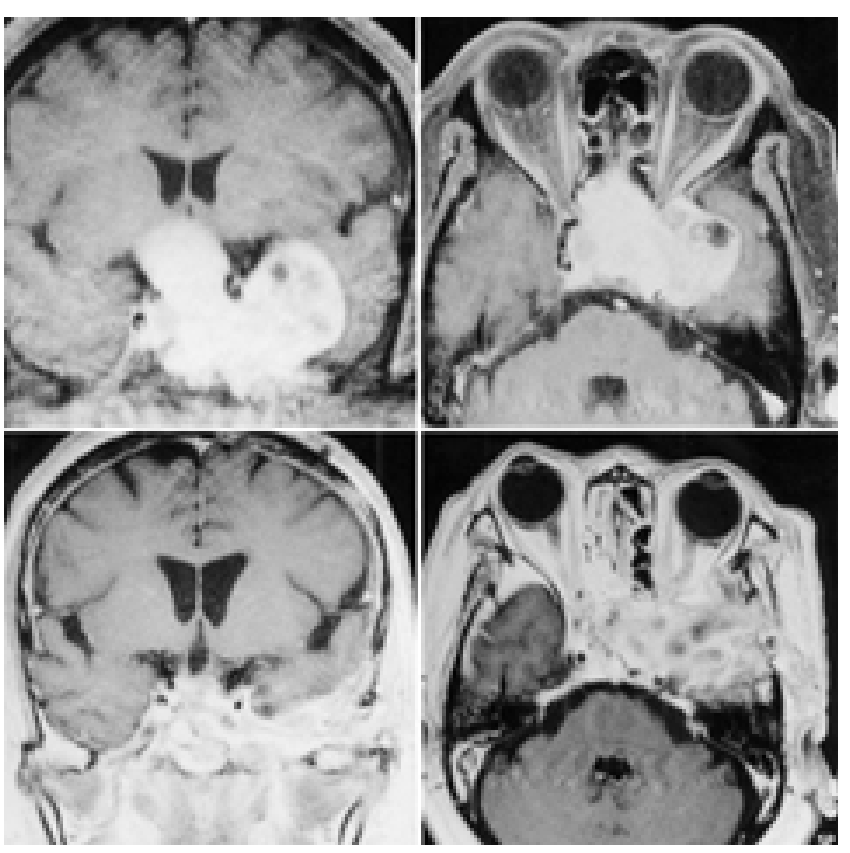

Fig. 3. Case 2. Gadolinium-enhanced MR images. Upper Left: Preoperative coronal image revealing a large chordoma in the sphenoid sinus, the medial temporal region, and suprasellar region. Upper Right: Preoperative axial image demonstrating bilateral cavernous sinus invasion. The left ICA is completely encased by the tumor. Lower Left: Postoperative coronal image revealing no residual tumor. Lower Right: Postoperative axial image demonstrating fat tissue in the operative fields and temporalis muscle. 


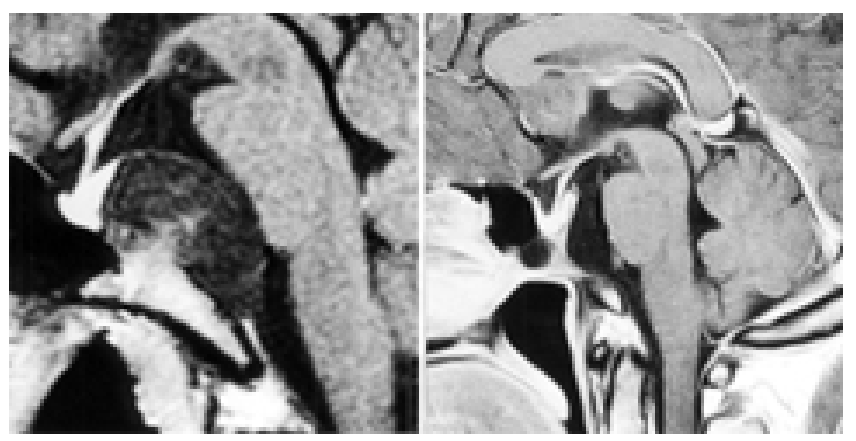

Fig. 4. Case 3. Gadolinium-enhanced MR images. Left: Preoperative sagittal image revealing a large chordoma in the upper and middle parts of the clivus. Right: Postoperative sagittal image demonstrating no tumor recurrence 7 years after the operation.

patient suffered no neurological deficits except for a residual hearing disturbance that proved to be due to an otological abnormality. No tumor recurrence was observed 7 years after the operation (Fig. 4 right).

\section{Case 4}

This 28-year-old woman who suffered from neck pain and left hand numbness was found to have a tumor at the CCJ. The tumor had invaded the lower clivus, the bilateral occipital condyles, the atlas, and the axis (Fig. 5 upper left and right). She underwent near-total resection of the tumor via the transcondylar approach (Fig. 5 center left and right) followed by occipitocervical fixation in which an autologous iliac bone graft was placed (Fig. 5 lower left). Postoperatively she underwent conventional radiotherapy (40 Gy). Follow-up MR imaging 3 years postoperatively revealed a minor recurrence of the tumor in the axis (Fig. 5 lower right); stereotactic radiosurgery was performed, with a maximum tumor dose of $23 \mathrm{~Gy}$ and a tumor margin (50\% isodose) dose of $12 \mathrm{~Gy}$. The patient has not shown any sign of tumor recurrence for 3 years after the radiosurgery.

\section{Case 5}

This 31-year-old woman underwent subtotal removal of a parasellar chordoma. The tumor had invaded the leftsided cavernous sinus. Conventional radiotherapy (60 Gy) was undertaken after the operation. Good local control of the tumor was shown, and the patient had been well without visual or ocular abnormalities until follow-up MR imaging at 18 years postoperatively revealed recurrence of the tumor; however, there was no evidence of neurological change. The patient underwent stereotactic radiosurgery (20 Gy maximum; $10 \mathrm{~Gy}$ at the $50 \%$ isodose line with 12 radiation isocenters). The patient remained asymptomatic, and no tumor growth was observed on MR imaging 3 years after the radiosurgery.

\section{DISCUSSION}

The management of chordomas and chondrosarcomas involving the cranial base remains controversial. ${ }^{3,5,7,17,21}$, 24,31 The therapeutic options are partial, subtotal, and radical resection, as well as various forms of radiotherapy. Both radical resection and stereotactic radiosurgery or

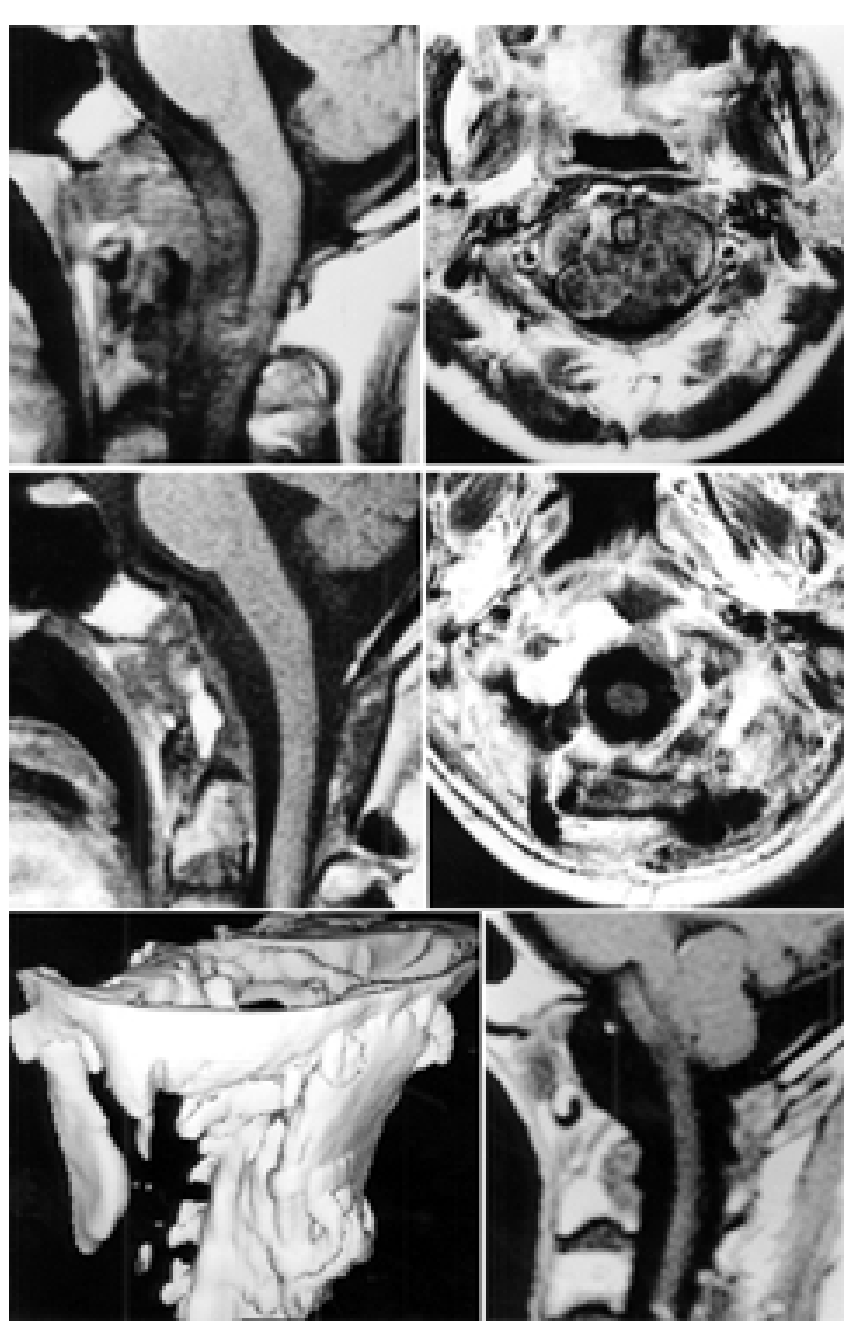

Fig. 5. Case 4. Gadolinium-enhanced MR images and CT study. Upper Left: Preoperative sagittal image revealing a large chordoma in the lower clivus, atlas, and axis. Upper Right: Preoperative axial image demonstrating invasion of the bilateral condyles. Center Left: Postoperative sagittal image revealing no residual tumor and good fixation of the CCJ. Center Right: Postoperative axial image demonstrating no residual tumor. Lower Left: Postoperative three-dimensional CT scan revealing good occipitocervical fusion with an iliac bone graft. Lower Right: Follow-up sagittal image obtained 3 years after the operation, demonstrating a recurrent tumor in the axis.

charged-particle irradiation have been reported to be effective for tumor control and better QOL. $3,8,13,17,20$

\section{Survival Rate}

Forsyth, et al., ${ }^{15}$ reported a review of 51 patients with intracranial chordomas surgically treated over a 24-year period. The 5-year survival rate for the 11 patients in whom biopsy samples were obtained was $36 \%$, whereas it was $55 \%$ for those who underwent subtotal resection. The authors drew several conclusions: the prognosis for patients with chordoma is unfavorable; young age is the single factor most strongly associated with longer survival; resection is beneficial; and postoperative radiotherapy may prolong disease-free survival. 
Gay, et al., ${ }^{17}$ reported the surgery-related results and follow up of 60 patients with cranial base chordomas and chondrosarcomas (median follow up 46.8 months). A variety of skull base approaches was used. Staged operations with combined approaches were performed in 52\% of the patients to resect the tumor more completely. Total or near-total resection was achieved in $67 \%$, subtotal in $23 \%$, and partial resection in $10 \%$ of the patients. The RFS rate for all patients was $76 \%$ at 5 years, and for those with chordoma it was $65 \%$ at 5 years. Patients with chondrosarcomas had a better prognosis than those with chordomas. In patients in whom total or near-total resection was achieved, the 5-year RFS rate (84\%) was better than in those in whom partial or subtotal resection was achieved $(64 \%)$. The authors pointed out that previous radiotherapy increased the risk of death during the postoperative and follow-up periods. Postoperative leakage of CSF was the most frequent procedure-related complication (30\% of the patients) and was found to increase the risk of permanent neurological deficits. For this reason, removal of patients' intra- and extradural tumor has recently been performed in two separate sessions. They concluded that total or near-total resection did not increase the rate of postoperative disability.

Over a 5-year period, Al-Mefty, and colleagues ${ }^{2}$ treated 25 patients who harbored skull base chordomas. They proposed a surgery-related classification system for skull base chordomas based on patterns and extension of the tumors. Radical resection was achieved in $10(43.5 \%)$ of 23 surviving patients, subtotal resection in 11 (47.8\%), and partial resection in two patients $(8.7 \%)$. Radical surgical removal included not only excision of soft-tumor tissue but also extensive drilling of the adjacent bone. Adjuvant therapy consisted of postoperative combined proton-photon beam radiotherapy in 17 patients and conventional radiotherapy in two. One patient who had undergone previous surgery and in whom the ICA was sacrificed died postoperatively of a massive stroke, and two patients died of tumor recurrence. Permanent neurological complications occurred in two patients. At the last reported follow-up study (mean 25.4 months), $71.4 \%$ of the patients were alive without evidence of tumor. The use of skull base approaches based on the tumor classification introduced in their report was credited with achieving the low mortality and morbidity rates, and they strongly advocated radical resection.

In our series, the overall 5-year RFS rate was 51\%, which is lower than that for other recently reported series. However, the RFS rate for patients who underwent surgery during the last decade was $77 \%$, which compares favorably with that reported in recent series. ${ }^{2,5,15,17}$ Total and near-total removal was achieved recently in four patients with acceptably low morbidity and no deaths. All of them survived with QOL during a median follow-up period of 5.3 years, although one recurrent tumor was found in a patient who subsequently underwent stereotactic radiosurgery for local tumor control. We therefore also concluded that for the treatment of skull base chordomas radical resection is a key factor longer survival and improved QOL.

\section{Surgical Treatment}

A variety of skull base approaches has been used to resect chordomas. Al-Mefty and associates ${ }^{3}$ reported on 17 patients who underwent one skull base procedure and six $(26 \%)$ who underwent two skull base procedures, and Gay, et al., ${ }^{17}$ reported that multistaged operations were conducted in $52 \%$ of their patients. We used a combined approach in four $(24 \%)$ and two-staged operations in two $(12 \%)$ of our patients mainly because of the presence of intradural invasion and multidirectional extension. In cases of extradural chordomas, careful attention should be paid to avoid damaging the dural barrier during resection to prevent intradural invasion by recurrent tumors. Treatment of chordomas with intradural invasion is very hazardous. Gay, et al., reported a high incidence of CSF leakage $(30 \%)$, which lead to a high incidence of meningitis $(10 \%)$. In our series, a postoperative CSF leak developed in one patient who had undergone a third operation for a recurrent tumor. To prevent CSF leakage, the defect of the skull base dura was repaired by performing a watertight reconstruction in which a fascial patch was reinforced by a vascularized flap and fibrin glue. If primary reconstruction is difficult, a two-stage operation should be selected.

\section{Selection of Approaches}

Transbasal Approach. The transbasal approach is used for tumors located in the midline that involve the sphenoethmoidal area and the upper and middle clivus. An extended frontal approach, which is a modification of the transbasal approach with additional bilateral orbitofrontal or orbitofrontoethmoidal osteotomy, allows the surgeon to reach the foramen magnum while minimizing the extent of the frontal lobe retraction. ${ }^{14,22,34}$ This route can be combined with other approaches such as a transmaxillary or transoral approach. The complication of anosmia caused by bilateral olfactory denervation is a disadvantage of this approach, but a circumferential osteotomy around the cribriform plate can preserve olfactory function. ${ }^{38}$ The lateral extension of this approach is limited by the optic nerves, cavernous sinuses, petrous apexes, abducent nerves, and hypoglossal canals. Combination with the lateral approach is necessary for tumors extending beyond these anatomical limits. We performed the transbasal approach in one patient (Case 1) with a chordoma that extended to the frontal base, the sphenoid sinus, and the middle part of the clivus. An alternative approach in this case is the transsphenoethmoidal approach.

Frontoorbitozygomatic Approach. The frontoorbitozygomatic approach is used for tumors located in the upper clivus and extending laterally into the cavernous sinus and intradural structures. Several advantages are associated with this approach: minimal brain retraction, a short distance to the lesions, multidirectional view, and an absence of contamination. ${ }^{1,3,35,36}$ In our series, this approach was used in conjunction with the transsphenoidal approach in cases in which there was cavernous sinus invasion and paraclinoid intradural extension, such as in Case 2. The disadvantage of this approach is the limited visualization it provides and the need to remove the extension of the tumor into the skull base bone, which may then require a second approach such as the transsphenoidal or the extended frontal approach.

Subtemporal-Infratemporal Approach. The subtem- 
poral-infratemporal approach provides a shorter and wider access to mid-skull base lesions than other approaches, regardless of whether the tumor is primarily extradural or both intra- and extradural. ${ }^{12,18,33}$ This approach can be combined with other lateral approaches such as the transpetrosal approach to access a clival tumor that extends laterally to the petrous bone. Although we have not used this approach in patients with chordomas, Gay, et al., ${ }^{17}$ have performed surgery via the subtemporal-infratemporal approach in $21 \%$ of their patients, and Al-Mefty and colleagues ${ }^{3}$ have reported using the zygomatic-extended middle fossa approach in $16 \%$ of their patients.

Transcondylar Approach. The transcondylar approach is used for tumors involving the lower clivus and the CCJ. ${ }^{37}$ This approach is especially useful for tumors that extend laterally to the CCJ. A short and wide operative field that avoids contamination is the advantage of this approach. ${ }^{9,37}$ Craniocervical stabilization can be achieved in the same session after resection of tumors invading the occipital condyles. Although the use of unilateral craniocervical fixation has been reported, ${ }^{2,37}$ we prefer midline occipitocervical fixation in which an autologous iliac bone graft is used in conjunction with a halo vest or instrumentation.

Transmaxillary Approach. The transmaxillary approach is particularly suitable for extradural lesions extending into the sphenoid and the upper and middle clivus with minimal lateral extension. ${ }^{11,16,30,39}$ Intradural tumors may be removed via the transmaxillary approach, but we prefer the lateral approach for those with intradural extension. The lateral boundaries are defined by the medial pterygoid plates, the ICAs at the level of the foramen magnum, the cavernous sinus, hypoglossal canals, and the jugular foramen. ${ }^{3,10}$ In addition to the LeFort I maxillotomy approach, the extended maxillotomy approach accompanied by a midline sagittal split of both maxilla and the soft palate facilitates access to the CCJ. ${ }^{11,21,23}$ In our series, one patient in whom total resection was achieved via by the transmaxillary approach has enjoyed RFS for more than 7 years without any complications. As an alternative to the transmaxillary approach, it should be determined whether a combined transoral-transsphenoidal approach will be adequate. The extended transsphenoidal ${ }^{3}$ - or transsphenoethmoidal approach incorporating an osteotomy of the frontal process of the maxilla ipsilateral to the lesion - can be used for tumors involving the upper clivus, sella turtica area, or the medial part of the cavernous sinus. ${ }^{3,25,26}$ The transoral approach is only suitable for extradural tumors at the ventral CCJ with minimal lateral extension. In our series, only partial or subtotal removal could be achieved using the transoral approach.

\section{Radiotherapy and Radiosurgery}

Most patients undergo postoperative radiotherapy in an attempt to control residual or recurrent tumors., $3,7,8,17,32$ With conventional irradiation modalities such as cobalt60 , only a moderate dose can be administered to skull base chordomas because of possible damage to the optic nerves and the brainstem. ${ }^{20}$ Because of this, tumor recurrence usually results. ${ }^{6}$

High-dose proton irradiation has excellent potential for achieving lasting tumor control and patient survival. Aus-
tin-Seymour, et al., ${ }^{7}$ have described 68 patients who underwent postoperative high-dose proton beam radiotherapy (median tumor dose of $69 \mathrm{GyE}$ ) and were followed for 17 to 152 months. The 5-year actuarial local control rate was $82 \%$ and the RFS rate was $76 \%$. They reported a better prognosis for small tumors and males. Munzenrider, et al. ${ }^{27}$ found that after radiation doses ranging from 56.8 to $75.6 \mathrm{cGyE}$, the 5-year actuarial local RFS rate was $76 \%$ and that the overall survival rate was $90 \%$ for all their 194 patients. Hug, et al., ${ }^{20}$ have described 58 patients who underwent proton beam radiotherapy for skull base chordoma and chondrosarcoma with target doses ranging from 64.8 to 79.2 cGyE (mean 70.7 cGyE). The 5-year actuarial local tumor control rates were $75 \%$ for chondrosarcoma and $59 \%$ for chordoma. Tumor volume and brainstem involvement influenced the control rate.

At present, the role of stereotactic radiotherapy as compared with particle beam radiotherapy, for the treatment of chordoma is not yet sufficiently clear. ${ }^{13,24}$ Recently, Muthukumar, et al., ${ }^{28}$ reported the use of gamma knife surgery in 15 patients with skull base chordoma or chondrosarcoma as an adjunct to or as an alternative to microsurgical resection. A maximum tumor dose of 24 to $40 \mathrm{~Gy}$ (mean $36 \mathrm{~Gy}$ ) and a tumor margin dose of 12 to 20 Gy (mean $18 \mathrm{~Gy}$ ) was administered to a mean tumor volume of $4.6 \mathrm{ml}$. Eight patients improved, three remained stable, and four died. Although actuarial survival rates were not reported the authors indicated that radiosurgery can be safely and efficiently administered to patients with small-volume tumors. Debus, et al., ${ }^{13}$ have reported that respective 5-year local control rates and survival rates were $50 \%$ and $82 \%$ for 45 cases of skull base chordomas or chondrosarcomas in which the patients had undergone fractionated stereotactic radiotherapy. In the series of patients who underwent proton radiotherapy reported by Hug, et al., ${ }^{20}$ most tumors exceeded the sizes reportedly suitable for stereotactic radiotherapy or they featured highly irregular configurations. Patients with tumors sufficiently small and with favorable configuration and location can be good candidates for stereotactic radiosurgery. Radical resection to reduce the tumor volume and to obtain an appropriate distance from the optic nerves, the chiasm, and the brainstem is the most important factor when using radiosurgery in the current multidisciplinary approach to the skull base chordomas.

\section{References}

1. Al-Mefty O, Anand VK: Zygomatic approach to skull-base lesions. J Neurosurg 73:668-673, 1990

2. Al-Mefty O, Borba LAB: Skull base chordomas: a management challenge. J Neurosurg 86:182-189, 1997

3. Al-Mefty O, Borba LAB, Aoki N, et al: The transcondylar approach to extradural nonneoplastic lesions of the craniovertebral junction. J Neurosurg 84:1-6, 1996

4. Amendola BE, Amendola MA, Oliver E, et al: Chordoma: role of radiation therapy. Radiology 158:839-843, 1986

5. Arnold H, Herrmann HD: Skull base chordoma with cavernous sinus involvement. Partial or radical tumor-removal? Acta Neurochir 83:31-37, 1986

6. Austin JP, Urie MM, Cardenosa G, et al: Probable causes of recurrence in patients with chordoma and chondrosarcoma of the base of skull and cervical spine. Int J Radiat Oncol Biol Phys 25:439-444, 1993 
7. Austin-Seymour M, Munzenrider J, Goitein M, et al: Fractionated proton radiation therapy of chordoma and low-grade chondrosarcoma of the base of the skull. J Neurosurg 70: 13-17, 1989

8. Berson AM, Castro JR, Petti P, et al: Charged particle irradiation of chordoma and chondrosarcoma of the base of skull and cervical spine: the Lawrence Berkeley Laboratory experience. Int J Radiat Oncol Biol Phys 15:559-565, 1988

9. Canalis RF, Martin N, Black K, et al: Lateral approach to tumors of the craniovertebral junction. Laryngoscope 103: 343-349, 1993

10. Catalano PJ, Biller HF, Sachdev V: Access to the central skull base via a modified Le Fort I maxillotomy: the palatal hinge flap. Skull Base Surg 3:60-68, 1993

11. Cocke EW, Robertson JH, Robertson JT, et al: The extended maxillotomy and subtotal maxillectomy for excision of skull base tumors. Arch Otolaryngol Head Neck Surg 116:92-104, 1990

12. Day JD, Fukushima T, Giannotta SL: Microanatomical study of the extradural middle fossa approach to the petroclival and posterior cavernous sinus region: description of the rhomboid construct. Neurosurgery 34:1009-1016, 1994

13. Debus J, Schulz-Ertner D, Schad L, et al: Stereotactic fractionated radiotherapy for chordomas and chondrosarcomas of the skull base. Int J Radiat Oncol Biol Phys 47:591-596, 2000

14. Derome PJ: Surgical management of tumors invading the skull base. Can J Neurol Sci 12:345-347, 1985

15. Forsyth PA, Cascino TL, Shaw EG, et al: Intracranial chordomas: a clinicopathological and prognostic study of 51 cases. $\mathbf{J}$ Neurosurg 78:741-747, 1993

16. Fraioli B, Esposito V, Santoro A, et al: Transmaxillosphenoidal approach to tumors invading the medial compartment of the cavernous sinus. J Neurosurg 82:63-69, 1995

17. Gay E, Sekhar LN, Rubinstein E, et al: Chordomas and chondrosarcomas of the cranial base: results and follow-up of 60 patients. Neurosurgery 36:887-897, 1995

18. Harsh GR, Sekhar LN: The subtemporal, transcavernous, anterior transpetrosal approach to the upper brain stem and clivus. J Neurosurg 77:709-717, 1992

19. Higinbotham NL, Phillips RF, Farr HW, et al: Chordoma Thirty-five-year study at Memorial Hospital. Cancer 20: 1841-1850, 1967

20. Hug EB, Loredo LN, Slater JD, et al: Proton radiation therapy for chordomas and chondrosarcomas of the skull base. J Neurosurgery 91:432-439, 1999

21. James D, Crockard HA: Surgical access to the base of skull and upper cervical spine by extended maxillotomy. Neurosurgery 29:411-416, 1991

22. Jane JA, Park TS, Pobereskin LH, et al: The supraorbital approach: technical note. Neurosurgery 11:537-542, 1982

23. Janecka IP, Sen CN, Sekhar LN, et al: Facial translocation: a new approach to the cranial base. Otolaryngol Head Neck Surg 103:413-419, 1990

24. Kondziolka D, Lunsford LD, Flickinger JC: The role of radio- surgery in the management of chordoma and chondrosarcoma of the cranial base. Neurosurgery 29:38-46, 1991

25. Lalwani AK, Kaplan MJ, Gutin PH: The transsphenoethmoid approach to the sphenoid sinus and clivus. Neurosurgery 31: 1008-1014, 1992

26. Laws ER Jr: Transsphenoidal surgery for tumors of the clivus. Otolaryngol Head Neck Surg 92:100-101, 1984

27. Munzenrider JE, Austin-Saymour M, Blitzer PJ, et al: Proton therapy at Harvard. Strahlentherapie 161:756-763, 1985

28. Muthukumar N, Kondziolka D, Lunsford LD, et al: Stereotactic radiosurgery for chordoma and chondrosarcoma: further experiences. Int J Radiat Oncol Biol Phys 41:387-392, 1998

29. O'Connell JX, Renard LG, Liebsch NJ, et al: Base of skull chordoma. A correlative study of histologic and clinical features of 62 cases. Cancer 74:2261-2267, 1994

30. Rabádan A, Conesa H: Transmaxillary-transnasal approach to the anterior clivus: a microsurgical anatomical model. Neurosurgery 30:473-482. 1992

31. Raffel C, Wright DC, Gutin PH, et al: Cranial chordomas: clinical presentation and results of operative and radiation therapy in twenty-six patients. Neurosurgery 17:703-710, 1985

32. Rich TA, Schiller A, Suit HD, et al: Clinical and pathologic review of 48 cases of chordoma. Cancer 56:182-187, 1985

33. Sekhar LN, Schramm VL, Jones NF: Subtemporal-peauricular infratemporal fossa approach to large lateral and posterior cranial base neoplasms. J Neurosurg 67:488-499, 1987

34. Sekhar LN, Nanda A, Sen CN, et al: The extended frontal approach to tumors of the anterior, middle, and posterior skull base. J Neurosurg 76:198-206, 1992

35. Sekhar LN, Ross DA, Sen C: Cavernous sinus and sphenocavernous neoplasms: anatomy and surgery, in Sekhar LN, Janecka IP (eds): Surgery of Cranial Base Tumors. New York: Raven Press, 1993, pp 521-604

36. Sekhar LN, Sen C, Synderman CH, et al: Anterior, anterolateral, and lateral approaches to extradural petroclival tumors, in Sekhar LN, Janecka IP (eds): Surgery of Cranial Base Tumors. New York: Raven Press, 1993, pp 157-223

37. Sen CN, Sekhar LN: An extreme lateral approach to intradural lesions of the cervical spine and foramen magnum. Neurosurgery 27:197-204, 1990

38. Spetzler RF, Herman JM, Beals S, et al: Preservation of olfaction in anterior craniofacial approaches. J Neurosurg 79: 48-52, 1993

39. Uttley D, Moore A, Archer DJ: Surgical management of midline skull-base tumors: a new approach. J Neurosurg 71: 705-710, 1989

Manuscript received January 16, 2001.

Accepted in final form February 8, 2001.

Address reprint requests to: Norihiko Tamaki M.D., Department of Neurosurgery, Kobe University School of Medicine, Kusunokicho, Chuo-ku, Kobe 650-0017 Japan. email: nagasima@ kobe-u.ac.jp. 